\title{
Guest Editorial: Clinical Ethics Consultation
}

\author{
Sumytra Menon $^{1} \cdot$ Marin Gillis $^{2}$
}

Published online: 23 March 2018

(C) National University of Singapore and Springer Nature Singapore Pte Ltd. 2018

This special issue celebrates the 13th International Conference on Clinical Ethics Consultation (ICCEC), which took place from 25 to 27 May 2017 in Singapore. The conference theme, Clinical Ethics and Change in Healthcare, asked presenters to contextualize clinical ethics support needs and meet challenges brought on by the evolving and changing healthcare landscape, including demographic shifts, biomedical advancements, system reforms, patient engagement, and an increased global interconnectivity. The conference sub-themes offered a chance for presenters to sharpen their focus on Clinical Ethics Support for Changing Health Care Practices and Contexts, Changing Attitudes to End-of-Life Care, Using Innovative Treatments and Modes of Health Service Delivery, and Globalization, Migration, and Cross-border Health Care. More than 310 leading clinicians, academics, policy-makers, and students from 38 countries answered the call to come to Singapore to exchange their work and deliberate and develop new global understandings. The papers in this issue are based on the presentations or are thematically aligned with ICCEC 2017.

The first paper in this special issue is the Asian Bioethics Review (ABR) First Prize winner, "A doctor in the house: Ethical and practical issues when doctors treat themselves and those they are close to," written by Kanny Ooi. This original article highlights the pitfalls of doctors treating themselves or those they are close to and compare the contrasting positions taken by the New Zealand and Singaporean regulators. The next paper is the ABR Second Prize winner, "How stigma distorts justice: The exile and isolation of leprosy patients in Hawai'i." The author, Alex Cheung, focuses on the ethical issues arising from the decision to exile patients with leprosy to a fortress island. "Using movie clips to promote reflective practice: A creative approach for teaching" is a perspective by Pablo G. Blasco, Graziela Moreto, and Leo Pessini that relates their experiences of using this pedagogical methodology to develop a more

Sumytra Menon

sumy.menon@nus.edu.sg

1 Centre for Biomedical Ethics, Yong Loo Lin School of Medicine, National University of Singapore, Singapore

2 Herbert Wertheim College of Medicine, Florida International University, Miami, FL, USA 
holistic approach to ethics education. Yap Su Yan presents a case report, "Use of the welfare-based model in the application of palliative sedation," which challenges the need for informed consent from the patient and instead promotes the welfare-based model as an alternative means of determining whether palliative sedation should be provided to a patient. Virginia Bartlett and Stewart Finder have written a paper highlighting the importance of patient preferences and values in promoting autonomous healthcare decision-making and the shift from advance directives in "An actual advance in advance directives: moving from patient choices to patient voices in advance care planning." In contrast, Mark Tan's perspective, "Considerations for Introducing Legislation on Advance Decisions in Malaysia" considers the challenges in healthcare decision-making for patients who lack capacity and those nearing the end of life when there are no relevant laws and ethical codes and guidelines are vague. Owen Schaefer in "Presenters or patients? A crucial distinction in individual health assessments" critiques a 2016 paper by Malone et al., which aimed to distinguish between patients, who receive regular medical care and presenters, which are asymptomatic but have undergone diagnostic health screening tests. Hui Jin Toh and co-authors have written the original article "Jonsen's Four Topics Approach as the Framework for Clinical Ethics Consultation." They reviewed the case records and other referral documentation of 28 patients referred to a clinical ethics committee over a 2-year period and conducted a thematic analysis offering unique insights into how their committee operates.

The papers accepted for this special issue undoubtedly satisfied the main theme of ICCEC 2017 but the focus was on the first three sub-themes, and we did not receive submissions on Using Innovative Treatments and Modes of Health Service Delivery, and Globalization, Migration, and Cross-border Health Care. The accepted papers address the evolving nature of clinical ethics healthcare, and the ICCEC conference was an excellent platform for generating discussion and advancing a scholarship on these issues with a global perspective. 